INPLASY

PROTOCOL

To cite: Wang et al. The benefits of Qigong exercise for symptoms of fatigue: a protocol for systematic review and meta-analysis. Inplasy protocol 2020110133. doi: 10.37766/inplasy2020.11.0133

Received: 29 November 2020

Published: 29 November 2020

Corresponding author:

Dai Sun

hzszyysd@126.com

Author Affiliation:

Hangzhou Hospital of

Traditional Chinese Medicine

Support: China Qigong

Management Center.

Review Stage at time of this submission: Preliminary searches.

Conflicts of interest: The authors of this work have nothing to disclose.

\section{The benefits of Qigong exercise for symptoms of fatigue: a protocol for systematic review and meta-analysis}

\author{
Wang, R¹; Huang, X2; Wu, Y3; Sun, $D^{4}$.
}

Review question / Objective: To assess whether Qigong exercise is safe and effective in the management of fatigue caused by different primary diseases along with an assessment of which type of Qigong exercise has a higher frequency of utilization.

Condition being studied: A large number of people suffer from continuous fatigue, which is also one of the most frequent symptoms seen in primary medical care. Fatigue, if not effectively relieved, may have an adverse effect on the quality of life and treatment of patients. Qigong is a safe and comfortable exercise popular in China and among the Ethnic Chinese, and is a complementary therapy used to treat fatigue, such as cancer related fatigue and chronic fatigue syndrome. It appears promising but lacks convincing evidence; thus, necessitating further investigation.

INPLASY registration number: This protocol was registered with the International Platform of Registered Systematic Review and Meta-Analysis Protocols (INPLASY) on 29 November 2020 and was last updated on 29 November 2020 (registration number INPLASY2020110133).

\section{INTRODUCTION}

Review question / Objective: To assess whether Qigong exercise is safe and effective in the management of fatigue caused by different primary diseases along with an assessment of which type of
Qigong exercise has a higher frequency of utilization.

Condition being studied: A large number of people suffer from continuous fatigue, which is also one of the most frequent symptoms seen in primary medical care. Fatigue, if not effectively relieved, may have 
an adverse effect on the quality of life and treatment of patients. Qigong is a safe and comfortable exercise popular in China and among the Ethnic Chinese, and is a complementary therapy used to treat fatigue, such as cancer related fatigue and chronic fatigue syndrome. It appears promising but lacks convincing evidence; thus, necessitating further investigation.

\section{METHODS}

Participant or population: Patients with primary disease-related symptoms of fatigue will be included, regardless of the underlying disease, age, gender, education, ethnicity, and occupation. However, if the patients included in the literature experience serious complications, cognitive communication disorders, or are pregnant, the study will be excluded.

Intervention: Qigong-related exercises alone (any form of Qigong category, including Baduanjin, Yijinjing, and Wuqinxi) or Qigong combined with other treatments will be the main intervention in the observation group. There is no limit to the duration and frequency of therapy.

Comparator: There is no exclusion based on the comparator method for this review, and the patients may be treated with any type of control group including exercise, stretching, sham Qigong, waiting list control, or other treatments.

Study designs to be included: Randomized controlled trials (RCTs) with no limitations on blinding published in English or Chinese will be included.

Eligibility criteria: Randomized controlled trials (RCTs) on Qigong exercise for patients with primary disease-related symptoms of fatigue with no limitations on blinding published in English or Chinese will be included.

Information sources: The following electronic databases will be searched: PubMed, Web of Science, Embase, Cochrane Library, China Biology Medicine disc, China National Knowledge
Infrastructure, WanFang and VIP Data Knowledge Service Platform. Furthermore, reference lists of included literature and previous relevant reviews will be manually retrieved to further locate additional trials and identify additional articles that meet our inclusion criteria.

Main outcome(s): Medical scales, which are mainly used to evaluate the severity, duration, frequency, improvement and symptoms of fatigue related to the primary disease, will be accepted as the main types of outcome measures. (For example, Flinders Fatigue Scale, Functional Assessment of Chronic Illness TherapyFatigue, Visual Analogue Scale-Fatigue, Multidimensional Fatigue Inventory).

Additional outcome(s): The quality of life assessment scale for social well-being, physical functioning, and mental functioning.

Quality assessment / Risk of bias analysis: Two researchers will independently evaluate the quality of the selected studies according to the Cochrane Collaboration's tool for randomized controlled trials. Items will be assessed in three categories: Low risk of bias, unclear bias and high risk of bias. The following characteristics will be evaluated: Random sequence generation (selection bias), Allocation concealment (selection bias), Blinding of participants and personnel (performance bias), Incomplete outcome data (attrition bias), Selective reporting (reporting bias) and other biases resulting from these questions will be graphed and assessed using the Review Manager 5.4. Any disagreements will be resolved by discussion or with the help of the third author.

Strategy of data synthesis: Efficacy data which conform to the same outcomes in the populations will be synthesized and statistically analyzed in STATA software for Windows 10. Risk ratio (RR) with 95\% confidence intervals (Cls) will be utilized to dichotomous data. For continuous data measured on the same measurement, the mean difference and $95 \% \mathrm{Cl}$ will be used for effect evaluation, while the standard 
mean difference will be used for results measured on different measurements. Random effects models (weighted by the inverse of the variance) will be used. A continuity correction will also be used for cells with zero values. $P$ value $<0.05$ will be considered statistically significant.

Subgroup analysis: If we acquire sufficient data, we plan to perform the analysis on the following subgroups of interest: primary diseases, Qigong exercises type, and quality of studies.

Sensibility analysis: If there is sufficient data for analysis, the sensitivity analysis will be performed to identify the results of the meta-analysis by excluding each of the individual studies.

Language: English or Chinese-language.

Country(ies) involved: China.

Keywords: Fatigue; Qigong; Protocol, Systematic review.

Contributions of each author:

Author 1 - Rui Wang.

Author 2 - Xueyan Huang.

Author 3 - Yeqi Wu.

Author 4 - Dai Sun. 